\title{
Probabilistic evaluation of snow-slab stability on mountain slopes
}

\author{
P. A. Chernouss, ${ }^{1}$ Yu.V. Fedorenko ${ }^{2}$ \\ ${ }^{1}$ Centre of Avalanche Safety, Production Association "Apatit”, 50th Anniversary of October Street, 184230 Kirovsk, Murmansk, Russia \\ ${ }^{2}$ Institute of Ecology, Kola Science Center, Russian Academy of Sciences, 35 Stroitelei Street, 184210 Apatity, Murmansk, Russia
}

\begin{abstract}
The results from a numerical-probabilistic experiment aimed at evaluating avalanche-release probabilities are presented in order to show the problems and the possibilities of such an approach. A snow slab on a mountain slope is modeled as a thin elastic non-moment shell resting on a three-dimensional hard underlying surface. The thickness of snow is considered as an inhomogeneous Gaussian spatial random field with a predetermined covariance that was obtained previously by field measurements. The stress distribution $\sigma(x, y)$ is calculated by solving the equations of equilibrium using a finite-difference method with a mesh of lines of curvature on a mountain slope. As a criterion of avalanche release, exceeding some threshold value of stress $\sigma_{\text {thres }}$ by the calculated value $\sigma(x, y)$ is applied. The field $P\left(\sigma(x, y)>\sigma_{\text {thres }} ; x, y\right)$ derived, using the Monte-Carlo method, is regarded as a measure of the spatial distribution of avalanche-release probability. This simulation scheme at present has no claim to forecast avalanche release but may be used to pinpoint the importance of snow distribution on a slope.
\end{abstract}

\section{INTRODUGTION}

Forecasting an avalanche hazard can be done by estimating the snow-cover stress field, which is related to snow thickness, density, shear and tensile properties and a dry-friction coefficient. If values of these parameters are known at any point on a snow slab, we can compute the stress field and determine potentially dangerous zones where the stress exceeds its threshold value.

Such a simple scheme is rarely applied to predicting an avalanche or to pinpointing dangerous zones in a deterministic manner. The reason is that the spatial variability of snowpack parameters is significant and, in practice, cannot be estimated with sufficient accuracy. This fact suggests the use of probabilistic methods, where the probability of density and covariance of snowpack parameters are used instead of their exact values. In previous work (Chernouss and Fedorenko, 1997), we undertook a numerical experiment considering snowpack thickness, cohesion and friction coefficients, and snow density as random values. It was not easy to interpret the results obtained because of the effect of multiple factors. In this study, we concentrate only on investigating the influence of random snowpack thickness to demonstrate the applicability of a probabilistic approach per se.

\section{PROBLEM FORMULATION}

The aim of our work is to determine snowpack stability on an arbitrarily shaped mountain slope, calculating the stress field within a three-dimensional snowpack. This is a very time-consuming task. However, as has been shown previously (Nefed'yev and Bozhinsky, 1989), the three-dimensional analysis may be reduced to a two-dimensional problem if the mechanical parameters of snow depend weakly on snow depth. In this case, the theory of equilibrium of thin shells can be applied.

The most appropriate coordinate system for this problem is a local orthogonal unit with vectors $\mathbf{e}_{1}, \mathbf{e}_{2}, \mathbf{e}_{3}$, where $\mathbf{e}_{1}$ and $\mathbf{e}_{2}$ are tangential to the two lines of curvature at any point of the surface and $\mathbf{e}_{3}=\mathbf{e}_{1} \times \mathbf{e}_{2}$ (Love, 1944). We assume that all points of the surface are non-umbilical and so at any point two distinct curvature lines exist. Under such assumptions, the stress field in the snowpack is governed by a simple system of partial differential equations expressing the equilibrium of a thin elastic shell (Nefed'yev and Bozhinsky, 1989);

$$
\begin{aligned}
\frac{\partial\left(h \sigma_{11}\right)}{\partial s_{1}}+\frac{\partial\left(h \sigma_{12}\right)}{\left.\partial s_{2}\right)}+\rho g h \mathbf{e}_{1} \mathbf{e}_{\mathrm{g}}-F_{\mathrm{fr}} \cos \alpha_{1} & =0, \\
\frac{\partial\left(h \sigma_{12}\right)}{\partial s_{1}}+\frac{\partial\left(h \sigma_{22}\right)}{\left.\partial s_{2}\right)}+\rho g h \mathbf{e}_{2} \mathbf{e}_{\mathrm{g}}-F_{\mathrm{fr}} \cos \alpha_{2} & =0, \\
N-h \sigma_{11} k_{1}-h \sigma_{22} k_{2}+\rho g h \mathbf{e}_{3} \mathbf{e}_{\mathrm{g}} & =0, \\
F_{\mathrm{fr}} & =c+f N
\end{aligned}
$$

where $\mathbf{e}_{\mathrm{g}}$ is a unit vector representing the gravitational force direction, $s_{1}, s_{2}$ the curvature coordinates, $h=h\left(s_{1}, s_{2}\right)$ the snowpack thickness measured perpendicularly to the slope surface, $\sigma_{i, j}$ the stress tensor, $i, j=1,2, \rho$ the snow density, $g$ the gravitational acceleration and $\cos \alpha_{1}$ and $\cos \alpha_{2}$ the directional cosines of the displacement vector $\mathbf{u}$. Note that $\cos \alpha_{1}=\cos \alpha_{2}$ if and only if $|\mathbf{u}|=\mathbf{0}$. $F_{\text {fr }}$ is the absolute value of the friction force between the snowpack and underlying surface, $c$ is the coefficient of cohesion, $f$ is the coefficient of friction, and $N$ is the normal pressure of the snowpack on the underlying surface. Equations (1) 
should be complemented by adding a system of linear equations which couples strains and stresses in the snowpack

$$
\begin{aligned}
\sigma_{11} & =\frac{E}{1-\nu^{2}}\left(\frac{\partial u_{1}}{\partial s_{1}}+\nu \frac{\partial u_{2}}{\partial s_{2}}\right), \\
\sigma_{12} & =\frac{E}{1+\nu}\left(\frac{\partial u_{1}}{\partial s_{2}}+\frac{\partial u_{2}}{\partial s_{1}}\right), \\
\sigma_{22} & =\frac{E}{1-\nu^{2}}\left(\frac{\partial u_{2}}{\partial s_{2}}+\nu \frac{\partial u_{1}}{\partial s_{1}}\right)
\end{aligned}
$$

where $E$ is the Young's modulus and $\nu$ is the Poisson ratio. According to Nefed'yev and Bozhinsky (1989) this system of equations must be solved with the Dirichlet boundary conditions $u_{\Gamma}=0$, where $\Gamma$ is the boundary of the snowpack surface.

In order to solve this set of equations, all snow parameters have to be specified. Many field experiments reveal a large spatial variability of the snow depth $h$ which suggests a stochastic description of this parameter. Other parameters, such as $\rho$ and $c$, can also be considered as random but their variability is usually less than the variability of $h$ (Föhn, 1989). Since Equations (1) contain derivatives of $h$, it is expected that the spatial random variation of $h$ will affect the solution more than the variations of $\rho$ and $c$. Specifically, in this study, the snow thickness is represented as an inho- mogeneous Gaussian spatial-random field with a predetermined covariance, which is obtained by field measurements.

The calculated displacement $\mathbf{u}$ and stress tensor $\sigma_{i, j}$, also become stochastic. Our aim is to find different statistical moments, like the probability $P\left(\sigma(x, y)>\sigma_{\text {thres }} ; x, y\right)$ to exceed some threshold value $\sigma_{\text {thres }}$ of stress at every point in the snowpack. The stochastic solution to the problem considered in this study is obtained by a Monte-Carlo simulation scheme. According to this scheme, Equations (1) and (2) are solved for a large number of realizations of $h\left(s_{1}, s_{2}\right)$. From the large number of deterministic solutions for these realizations, any desired statistical moment can be obtained.

\section{PREPROCESSING}

Because of the extreme requirement for computational speed, we chose a finite-difference technique for solving Equations (1) and (2) numerically. The first step was to generate a mesh of lines of curvature and build a random field of snowpack thickness with Gaussian distribution and prescribed covariance. As assumed, the slope surface $z=z(x, y)$, where $x, y, z$ are Cartesian coordinates, does

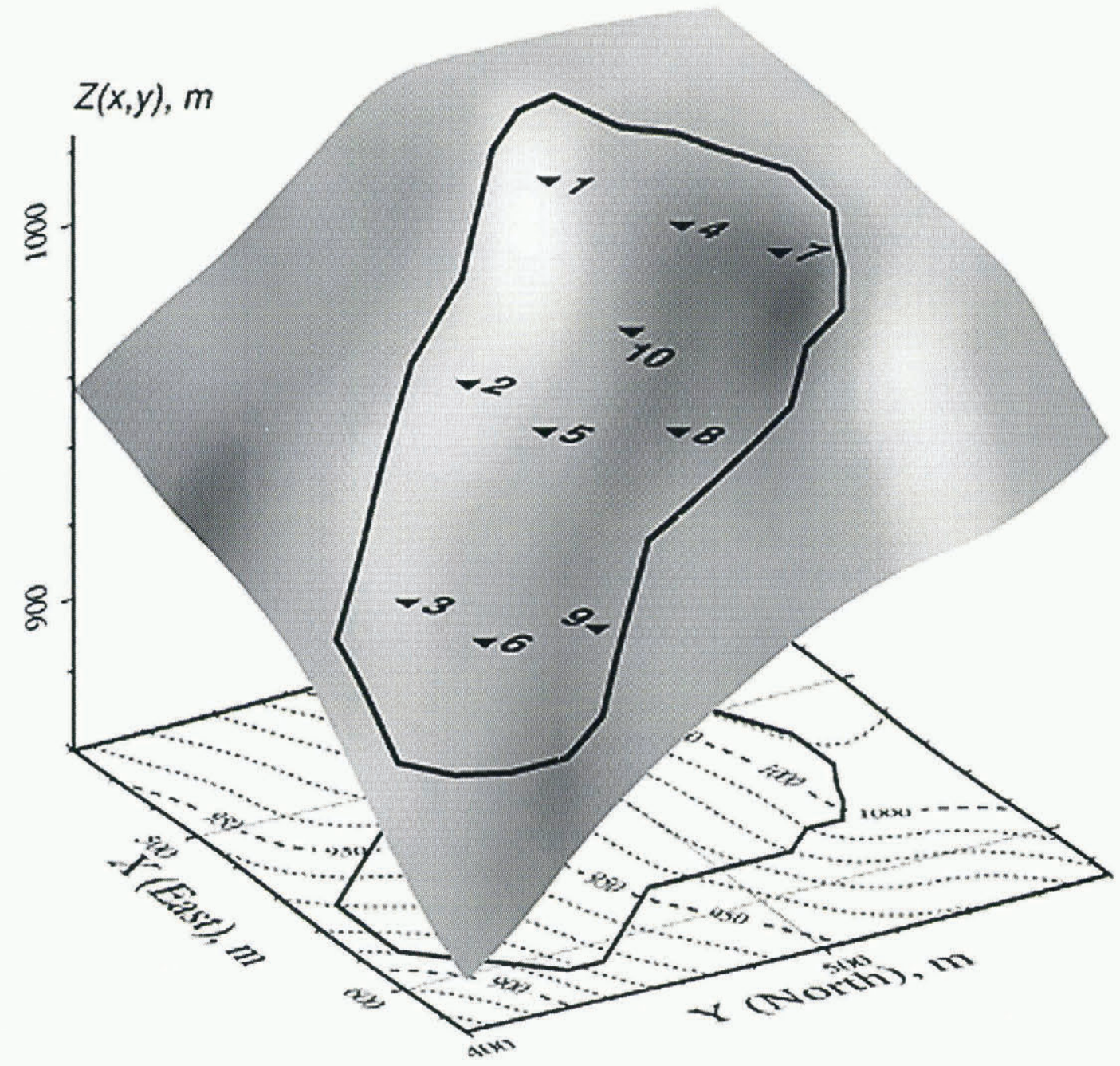

Fig. 1. The geometry of avalanche starting zone No.46 at the quarry of the "Apatit" 7.SC in Khibiny mountains, Kola Peninsula. Numbered triangles on the slope surface denote the points where the snow thickness has been observed. The thick solid line indicates the boundary $\Gamma$ where the condition $u_{\Gamma}=0$ is applied. 


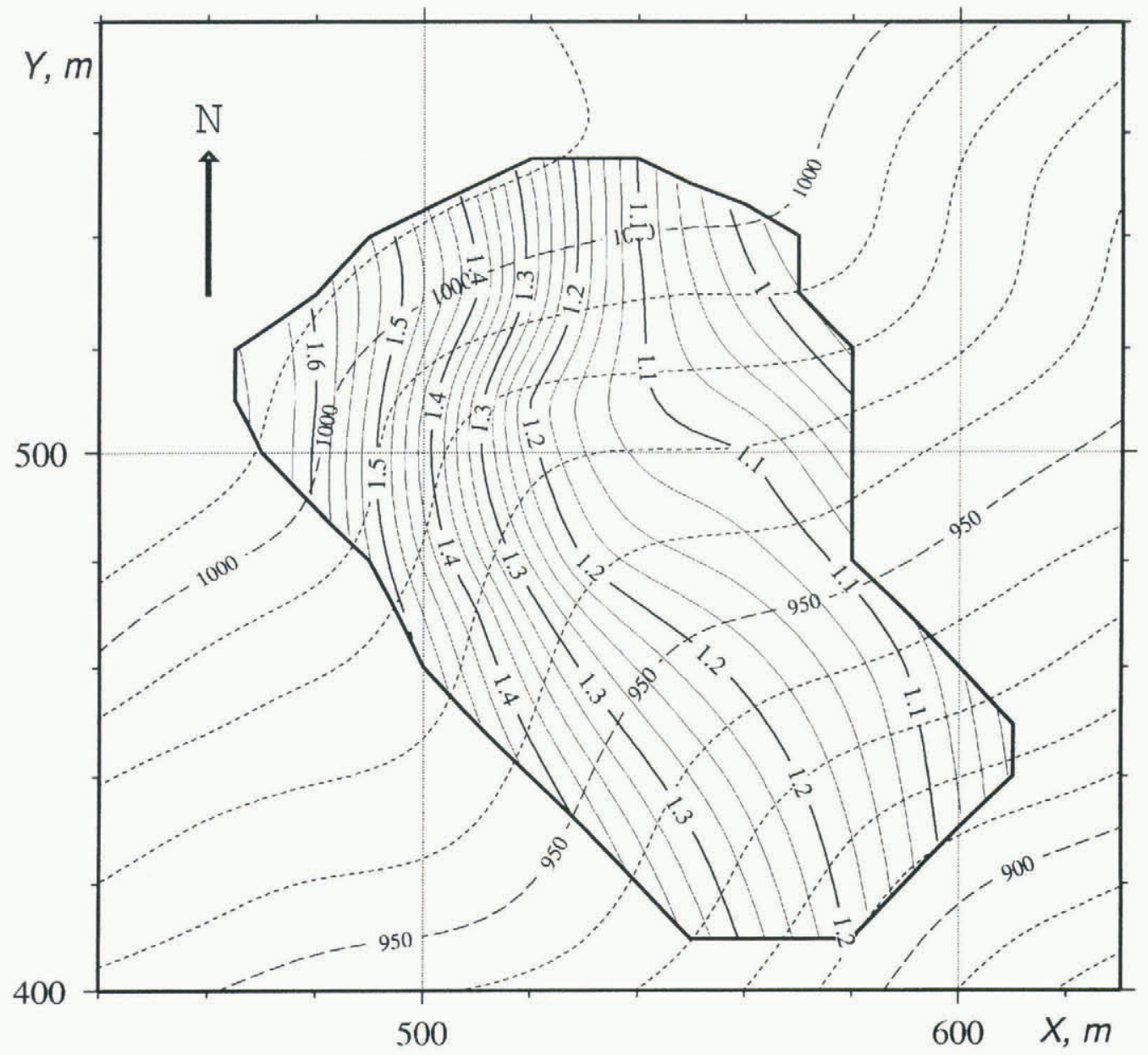

Fig. 2. Isolines of the regular term $\bar{h}(x, y)$ in $m$ of snow thickness (solid) and the steep-slope topography in $m$ a.s.l. (dashed). Slope angles vary from $20^{\circ}$ to $43^{\circ}$.

not contain umbilical points. The lines of curvature in this case may be found by solving the ordinary differential equations (Korn and Korn, 1968)

$$
\left|\begin{array}{ccc}
\mathrm{d} y^{2} & -\mathrm{d} x \mathrm{~d} y & \mathrm{~d} x^{2} \\
E & F & G \\
L & M & N
\end{array}\right|=0
$$

where

$$
\begin{aligned}
E=1+(\partial z / \partial x)^{2}, F & =(\partial z / \partial x)(\partial z / \partial y), G=1+(\partial z / \partial x)^{2}, \\
L & =\frac{\partial^{2} z / \partial x^{2}}{{\sqrt{1+(\partial z / \partial x)^{2}+(\partial z / \partial y)^{2}}}^{2}}, \\
M & =\frac{\partial^{2} z / \partial x \partial y}{{\sqrt{1+(\partial z / \partial x)^{2}+(\partial z / \partial y)^{2}}}^{2}}, \\
N & =\frac{\partial^{2} z / \partial y^{2}}{{\sqrt{1+(\partial z / \partial x)^{2}+(\partial z / \partial y)^{2}}}^{2}} .
\end{aligned}
$$

In practice, a mountain slope is represented as a discrete set of samples $\left(x_{\mathrm{i}}, y_{\mathrm{i}}, z_{\mathrm{i}}\right)$. To calculate the coefficients of $E$ to $N$ of Equation (3), the mountain slope in the vicinity of a possible avalanche starting zone should be interpolated or approximated, for example, by a cubic surface. The pair of Equations (3) may then be solved easily by the adaptive Runge-Cutta method (Press and others, 1992).

As assumed above, the fluctuating component of the snowpack thickness $\tilde{h}\left(s_{1}, s_{2}\right)$ has a normal distribution and covariance $\psi_{\mathrm{h}}\left(s_{1}, s_{2}\right)$, which is obtained from field measurements. The realization of the snowpack thickness $h\left(s_{1}, s_{2}\right)$ is a sum of regular $\bar{h}\left(s_{1}, s_{2}\right)$ and fluctuating $\tilde{h}\left(s_{1}, s_{2}\right)$ components, $h\left(s_{1}, s_{2}\right)=\bar{h}\left(s_{1}, s_{2}\right)+\tilde{h}\left(s_{1}, s_{2}\right)$. Here, we assume that the regular term varies much more slowly than the fluctuating one. The regular term may be found from direct measurements of snow thickness at the control points on the slope $H_{\mathrm{i}}, i=1, \ldots, M$, where $M$ is the number of control points on the considered slope. To build a random field with such properties we perform the following steps:

Generate the Gaussian $\delta$-correlated spatial random field $\tilde{h}_{\delta}\left(s_{1}, s_{2}\right)$ with zero mean and given variance.

Transform the generated random field $\tilde{h}_{\delta}\left(s_{1}, s_{2}\right)$ from the space domain to wave-number domain by the two-dimensional fast Fourier transform (Press and others, 1992).

Transform in a similar manner the covariance $\psi_{\mathrm{h}}\left(s_{1}, s_{2}\right)$ to its power spectra $\Psi_{\mathrm{h}}\left(k_{1}, k_{2}\right)$.

Fit $\bar{h}\left(s_{1}, s_{2}\right)$ to snow thickness data $H_{\mathrm{i}}$ obtained by direct measurements.

Build the fluctuating component $\bar{h}\left(s_{1}, s_{2}\right)$ in a space domain by inverse Fourier transform of the function $h_{\delta}\left(k_{1}, k_{2}\right) \sqrt{\Psi_{\mathrm{h}}\left(k_{1}, k_{2}\right)}$ and calculate the desired field $h\left(s_{1}, s_{2}\right)=\bar{h}\left(s_{1}, s_{2}\right)+\tilde{h}\left(s_{1}, s_{2}\right)$.

These operations yield a mesh of curvature lines and a random field of snow thicknesses conforming both with the existing covariance $\psi_{\mathrm{h}}\left(s_{1}, s_{2}\right)$ and measured values $H_{\mathrm{i}}$. 


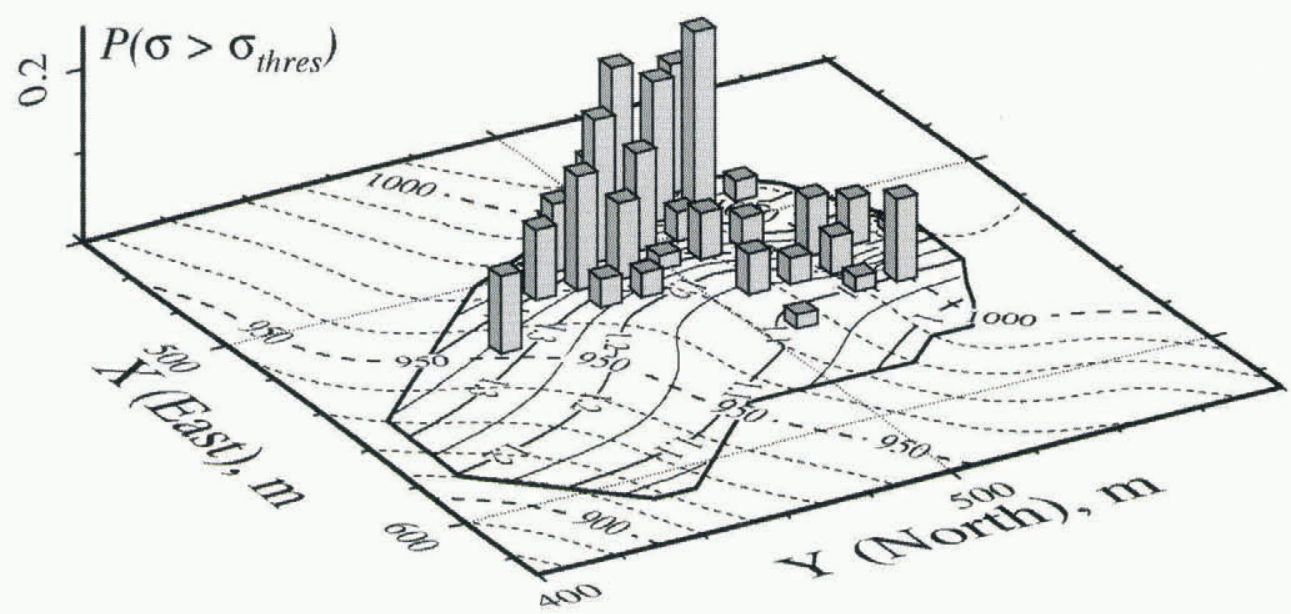

Fig. 3. Preliminary results of simulation. The columns present the spatial distribution of the probability $P\left(\sigma(x, y)>\sigma_{\text {thres }} ; x, y\right)$. The maximum value of probability is 0.21 , the minimum is 0.012 . The values of $P\left(\sigma>\sigma_{\text {thres }}\right)$ less than 0.01 are not shown.

\section{SOME RESULTS AND DISGUSSION}

The main goal of this study was to demonstrate the applicability of the probabilistic approach and to improve our understanding of stochastic behavior of the stress field in the snowpack with a random component of the thickness. The ultimate goal is to identify slopes which may be dangerous. For our simulation we took avalanche start-zone number 46 (Fig. 1) at the quarry of "Apatit" JSC in the Khibiny mountains, Kola Peninsula. The contoured part of the slope was substituted with a cubic surface, where the rms fitting error was about $2.1 \mathrm{~m}$. The grid of lines of curvature used in the finite-difference simulation was evaluated by the Runge-Kutta method (Press and others, 1992). The regular term of snow thickness (Fig. 2) was found by the program "surface" that is a component of generic mapping tools (GMT) software (Wessel and Smith, 1995). The fluctuating part of the snow thickness was calculated using the empirical covariance $\psi_{\mathrm{h}}(l)=\left[0.38 \bar{h}\left(s_{1}, s_{2}\right)\right]^{2} \exp \left(-0.09 l^{1.5}\right), l=\sqrt{s_{1}^{2}+s_{2}^{2}}$, obtained from long series of field measurements in Khibiny (Chernouss, 1995). We chose typical values of snow density $\rho=300 \mathrm{~kg} \mathrm{~m}^{-3}$, coefficient of cohesion $c=2000 \mathrm{~Pa}$, coefficient of friction $f=0.4$ and $\sigma_{\text {thres }}=2000 \mathrm{~Pa}, \nu=0.3$, $E=10^{7} \mathrm{~Pa}$.

The results of stochastic modeling are shown in Figure 3. The most important result of our simulations is that the probability of avalanche release depends on snow thickness. At the upper part of the avalanche start zone, where the snow slab is thickest, the excedance probability $P\left(\sigma>\sigma_{\text {thres }}\right)$ appears to be much larger whereas the slope geometry is quite similar. This result is expected and substantiates the validity of the method. Using our simulation method, it is possible to obtain other quantities, such as the density function of the probability of avalanche-release occurrence at any point on the slope. At present, it is beyond the scope of our work to forecast the time and place of avalanche release, even if this were feasible, since we do not have sufficient knowledge of the snow parameters.

Our experience testifies to the efficiency and good convergence of the finite-difference scheme on regular slopes that do not include umbilical points and lines of inflection. The demand for regularity may be a serious limitation of this scheme, since the surface of a real slope often contains singularities and hence numerical difficulties arise while building the model mesh. A possible way of avoiding this limitation is to use the finite-element method to solve Equations (1) and (2) numerically.

In order to introduce the proposed method for estimating avalanche-release probability for avalanche forecasts, it has to be verified beforehand using the available data. Since we obtain statistical values as an output of our simulation, the raw data should be statistically processed in order to obtain comparable data. The database and the necessary software are currently in preparation at the Centre of Avalanche Safety of the "Apatit"JSC.

\section{REFERENCES}

Chernouss, P. 1995. Spatial and time variability of avalanche predictors and accuracy of their estimations. In Sivardière, F., ed. Les apports de la recherche scientifique à la sécurité neige, glace et avalanche. Actes de Colloque, Chamonix 30 mai - 3 juin 1995. Grenoble, Association Nationale pour l'Étude de la Neige et des Avalanches (ANENA), 123-128.

Chernouss, P. A. and Yu.V. Fedorenko. 1997. The stochastic model of snow cover stability on mountain slopes. In ISSW'96. International Snow Science Workshop, 6-10 October 1996, Banff, Alberta. Proceedings. Revelstoke, B.C., Canadian Avalanche Association, 104-106.

Föhn, P. M. B. 1989. Snowcover stability tests and the areal variability of snow strength. In A Merging of Theory and Practice: Proceedings of the International Snow Science Workshop, 12-15 October 1988, Whistler, B.C. Vancouver, B.C., Canadian Avalanche Association, 262-273.

Korn, G. A. and T. M. Korn. 1968. Mathematical handbook for scientists and engineers. New York, McGraw-Hill Book Company.

Love, A. E. H. 1944. A treatise on the mathematical theory of elasticity. New York, Dover.

Nefed'yev, V. O. and A. N. Bozhinskiy. 1989. Ravanovesiye tonkoy vesomoy uprugoy obopochkina zhestkom osnovanii [Balance of thin weightable elastic shell on the hard base.] Dubna, Joint Institute of Nuclear Research. (Report P5-89-694.)

Press, W. H., B. P. Flannery, S. A. Teukolsky and W.T. Vetterling. 1992. Numerical recipes in $C:$ the art of scientific computing. Second edition. Cambridge, Cambridge University Press.

Wessel, P. and W. H.F. Smith. 1995. New version of the Generic Mapping Tools released. EOS, 76, 329. 